\title{
Erratum to: An estimation of ENSO predictability from its seasonal teleconnections
}

\section{Tasambay-Salazar ${ }^{1,2}$ • M. J. OrtizBeviá ${ }^{1}$ - F. J. Alvarez-García ${ }^{1}$.}

\section{A. M. RuizdeElvira ${ }^{1}$}

Published online: 7 August 2015

(C) Springer-Verlag Wien 2015

\section{Erratum to: Theor Appl Climatol}

DOI 10.1007/s00704-015-1546-3

The original version of this article unfortunately contained mistakes. Four of the Appendix equations were incorrect. The corrected equations are given below.

Old

$d_{t} \vec{y}_{j}^{s}+A \vec{y}^{s}(t)=\sum_{j=1}^{P} \vec{N}_{j}(t)$

New

$d_{t} \vec{y}^{s}(t)-A \vec{y}^{s}(t)=\sum_{j=1}^{p} \vec{N}_{j}(t)$

The online version of the original article can be found at http://dx.doi.org/ 10.1007/s00704-015-1546-3.

\section{J. OrtizBeviá}

mjose.ortiz@uah.es

Departamento de Física y Matemáticas, Universidad de Alcalá, 28801 Alcalá de Henares, Spain

2 Facultad de Informática y Electrónica, Escuela Superior Politécnica de Chimborazo, Riobamba, Ecuador
Old

$$
\begin{aligned}
\vec{y}^{s}(t+\Delta t) & =\vec{y}^{s}(t)+G(t+\Delta t) \vec{y}^{s}(t) \\
& +G(t+\Delta t) \int_{t}^{t+\Delta t} G^{-1}(\tau) \sum_{j} N_{j}(\tau) d \tau
\end{aligned}
$$

New

$$
\vec{y}^{s}(t+\Delta t)=G(\Delta t) \vec{y}^{s}(t)+G(t+\Delta t) \int_{t}^{t+\Delta t} G^{-1}(\tau) \sum_{j=1}^{p} \vec{N}_{j}(\tau) d \tau
$$

Old

$N_{j}(t)=\sum_{d=1}^{n} \vec{\psi}_{j d} \pi_{d} R_{d}(t)$

New

$\vec{N}_{j}(t)=\sum_{d=1}^{n} \vec{\psi}_{j d} \pi_{d} R_{d}(t)$

Old

$$
\begin{aligned}
\vec{y}^{s}(t+\Delta t)=\vec{y}^{s}(t)+G(t+\Delta t) \vec{y}^{s}(t)+G(t+\Delta t) \\
\int_{t}^{t+\Delta t} G^{-1}(\tau)\left(\sum_{j=1}^{p} \sum_{d=1}^{n} \vec{\psi}_{j d} \pi_{d} R_{d}(t) d \tau\right)
\end{aligned}
$$

New

$$
\begin{aligned}
\vec{y}^{s}(t+\Delta t)=G(\Delta t) \vec{y}^{s}(t)+G(t+\Delta t) \int_{t}^{t+\Delta t} \\
G^{-1}(\tau)\left(\sum_{j=1}^{p} \sum_{d=1}^{n} \vec{\psi}_{j d} \pi_{d} R_{d}(t) d \tau\right)
\end{aligned}
$$

